\title{
Jürgen Court
}

\section{Sportwissenschaft}

\begin{abstract}
„Ein verfaulter Körper wird durch einen strahlenden Geist nicht im geringsten ästhetischer gemacht, ja, es ließe sich höchste Geistesbildung gar nicht rechtfertigen, wenn ihre Träger gleichzeitig körperlich verkommene und verkrüppelte, im Charakter willensschwache, schwankende und feige Subjekte wären. [...] Wenn heute, selbst im Lehrplan der Mittelschulen, Turnen in einer Woche mit knappen zwei Stunden bedacht wird und die Teilnahme daran als nicht obligat dem einzelnen freigegeben wird, so ist dies, verglichen zur rein geistigen Ausbildung, ein krasses Mißverhältnis." 1
\end{abstract}

\section{Zur Kontinuitätsfrage der Geisteswissenschaft}

Dieses Zitat aus Hitlers „Kampfbuch“2, entnommen dem Kapitel „Erziehungsgrundsätze des völkischen Staates", steht deshalb gleich am Beginn dieses Beitrags, weil es berechtigte Zweifel an der Rechtmäßigkeit des Vorhabens weckt, über Sportwissenschaft im Dritten Reich als eine Geisteswissenschaft zu sprechen. Wenn wir die Wissenschaft vom Sport, sofern sie Geisteswissenschaft ist, als Theorie eines bestimmten Verhältnisses von "körperlichen" zu "geistigen" 3 Handlungen bestimmen, was bedeutet es dann für diese Wissenschaft, wenn - wie hier bei Hitler - dem, Geist' das Existenzrecht abgesprochen wird? Auch der Völkische Beobachter vom 13. August 1932 monierte, daß "unsere Sportführer" nur Abstrakta wie „Geist [!], Technik und Taktik“4 im Sinne hätten, und pikanterweise schlägt Theodor W. Adorno in die gleiche Kerbe: Der moderne Sport „äh-

1 Adolf Hitler, Mein Kampf (München 1937) 453f.

2 Victor Klemperer, LTI (Berlin 1947) 9; im folgenden zitiert: Klemperer, LTI.

3 Vgl. Wilhelm Benary, Der Sport als Individual- und Sozialerscheinung (Berlin 1913) 90f.: „Die Sonderung in körperlichen und geistigen Sport hat ihre Bedeutung darin, daß durch sie Sports unterschieden werden, in denen körperliche Übungen eine hervorragende Rolle spielen, und solche, in denen es nicht der Fall ist. Denn ein geistiges Element ist in jedem Sport vorhanden, rein körperliche Sports gibt es nicht." Ein wissenschaftstheoretischer Kommentar findet sich bei Jürgen Court, Kleine Ideengeschichte der Sportwissenschaft 1900-1914 (St. Augustin 1998) $161 \mathrm{ff}$; im folgenden zitiert: Court, Ideengeschichte.

${ }^{4}$ Zit. n. Hajo Bernett, Der Deutsche Olympische Ausschuß - das nationale olympische Komitee zur Vorbereitung auf die Olympischen Spiele 1928, 1932 und 1936, in: Sportwissenschaft 26 (1996) 145. 
nelt den Leib tendenziell selber der Maschine an. Darum gehört er ins Reich der Unfreiheit, wo immer man ihn auch organisiert" 5 .

Solche Antworten sind für uns jedoch wenig hilfreich. Sie verfehlen schlichtweg das Phänomen, sind ungeschichtlich und unterschlagen daher das entscheidende „Kontinuitätsproblem"6: die Frage nach der Kontinuität und Diskontinuität historischer Ereignisse. Es handelt sich in der Deutung von Hitlers Zitat nicht etwa um Existenz oder Nichtexistenz des Geistes einer bestimmten Wissenschaft, sondern um eine bestimmte Art und Weise der Interpretation dieses Geistes: „Es geht uns [...] nicht um einen Kampf gegen, sondern um den Geist, für den Geist, und wir vertreten unsererseits einen neuen Geistbegriff. “7

Mit dieser Korrektur rückt die Frage nach der Kontinuität und Diskontinuität der Sportwissenschaft im Nationalsozialismus in den geistesgeschichtlichen Horizont des „Idealismusparadigmas"8, mit dem in der Diktion der Kuhnschen Paradigmentheorie der in der Weimarer Republik ,in fast allen Wissenschafts-Disziplinen wirksame antipositivistische Reflex "9 bezeichnet wird. Obgleich die Einsicht in die Notwendigkeit, zur Erforschung des nationalsozialistischen Sports auch seine ideengeschichtlichen Voraussetzungen zu berücksichtigen, Allgemein-

5 Theodor W. Adorno, Das Reich der Unfreiheit und der Sport, in: Sportphilosophie, hrsg, v. Volker Caysa (Leipzig 1997) 43. Zur Kritik dieser „pauschalisierenden Totalitätsperspektive", in der sich die marxistische mit der nationalsozialistischen Ideologie berührt, vgl. Kurt Salamun, Kritische Gesellschaftstheorie und Sportkritik der Neuen Linken. Zur Diskussion um eine Philosophie und Soziologie des Sports, in: Aktuelle Probleme der Sportphilosophie, hrsg. v. Hans Lenk (Schorndorf 1983) 368ff.; Notker Hammerstein, Antisemitismus und deutsche Universitäten. 1871-1933 (Frankfurt a.M., New York 1995) 93, setzt die Disziplin der "Sportwissenschaften" in der Weimarer Republik distanzierend in Anführungszeichen; Winfried Joch, Sport und Leibeserziehung im Dritten Reich, in: Leibesübungen und Sport in Deutschland vom Ersten Weltkrieg bis zur Gegenwart, hrsg. v. Horst Ueberborst (Berlin 1982) 706 (im folgenden zitiert: Joch, Sport), benutzt die Anführungszeichen für den Sport im Nationalsozialismus. Bei Adolf Jürgens (Hrsg.), Ergebnisse deutscher Wissenschaft. Eine bibliographische Auswahl aus der deutschen wissenschaftlichen Literatur der Jahre 19331938 (Essen 1939) 496 ff., 586 f., wird der Begriff „Sportmedizin“ der Medizin und der Begriff

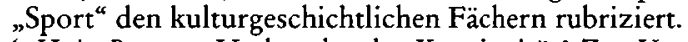

${ }^{6}$ Hajo Bernett, Umbruch oder Kontinuität? Zur Vorgeschichte des Deutschen Reichsbundes für Leibesübungen, in: Sozial- und Zeitgeschichte des Sports 9 (1995) 2, 7 f., Anm. 2; im folgenden zitiert: Bernett, Umbruch; vgl. Hans Joachim Teichler, Die Rolle Carl Diems in der Zeit und im zeitlichen Umfeld des NS-Regimes, in: Sozial- und Zeitgeschichte des Sports 10 (1996) 3, 56; im folgenden zitiert: Teichler, Die Rolle Carl Diems; zur Romanistik vgl. die programmatischen Thesen von Frank-Rutger Hausmann, Auch eine nationale Wissenschaft? Die deutsche Romanistik unter dem Nationalsozialismus, 1. Teil, in: Romanistische Zeitschrift für Literaturgeschichte 22 (1998) 4 ff.; im folgenden zitiert: Hausmann, Nationale Wissenschaft 1 . Teil.

7 Alfred Rosenberg, zit. n. Monika Leske, Philosophen im „Dritten Reich“ (Berlin 1990) 84; im folgenden zitiert: Leske, Philosophen.

8 Hausmann, Nationale Wissenschaft 1. Teil, 7.

${ }^{9}$ Hans Manfred Bock, Das Eigene, das Fremde und das Ganz-Andere - Zur Stellung Victor Klemperers in der kulturkundlichen Frankreich-Diskussion der Weimarer Republik, in: lendemains 21 (1996) 97; im folgenden zitiert: Bock, Zur Stellung Victor Klemperers. 
gut der Forschung ist, fehlte bislang eine solche systematische Analyse ${ }^{10}$. Diese Behauptung steht nicht im Gegensatz zu der These, daß die Zeit der NS-Diktatur „ein von der neueren bundesdeutschen Sportgeschichtsschreibung intensiv bearbeitetes Feld ist“" ${ }^{11}$, da sie sich lediglich auf die Dominanz „sozialgeschichtlicher Fragestellungen" 12 bezieht.

Weshalb hier die genauere Bestimmung dieser paradigmatischen „Schnittstelle" ${ }^{13}$ zwischen der Zeit vor und nach 1933 durch einen Vergleich zwischen dem Geistbegriff der Sportwissenschaft und dem einer Sprachwissenschaft, der Romanistik, geschieht, hat folgende Gründe. Erstens weist Hitlers eingangs zitierte Opposition vom „verfaulten Körper“ und „strahlenden Geist“ auf das in der Romanistik und Sportwissenschaft der Zwanziger Jahre am häufigsten benutzte und variierte Zitat: auf Friedrich Schillers idealistisches Credo „Es ist der Geist, der sich den Körper baut" ${ }^{* 14}$. Zweitens beschäftigt sich diese Studie vor allem mit Carl Diem und Victor Klemperer, weil sie dieses Zitat besonders häufig anführen und weil beide als Vertreter ihres Faches eine große, über den engeren Kreis der Wissenschaft hinausgehende Bekanntheit erreicht haben, die nicht zuletzt auf der öffentlichen Diskussion des Problems beruht, wie ihr persönliches und wissenschaftliches Schaffen vor der Folie ihres politischen und gesellschaftlichen Umfeldes angemessen beurteilt werden könne. Die Konzentration auf diese beiden Exponenten ihrer Wissenschaft ist vor dem Hintergrund jenes Zitats von Schiller deswegen geboten, weil ihre jeweilige Interpretation eng mit der Frage verknüpft ist, weshalb beide trotz des in der Weimarer Zeit identischen idealistischen Fundamentes nach 1933 so verschiedene Wege gehen. Und mit der Antwort auf sie hängt es wiederum zusammen, daß mit Klemperers kritischer Beobachtung des Sports im Nationalsozialismus Sport und Sportwissenschaft von zwei völlig verschiedenen Seiten her in den Blick genommen werden können.

10 Ansätze bei Ommo Grupe, Studien zur pädagogischen Theorie der Leibeserziehung (Schorndorf 21968) 37f.; im folgenden zitiert: Grupe, Pädagogische Theorie; Stefan Größing, Pädagogische Reformen vor und nach dem Ersten Weltkrieg und ihr Einfluß auf Leibeserziehung und Schulsport, in: Leibesübungen und Sport in Deutschland vom Ersten Weltkrieg bis zur Gegenwart, hrsg. v. Horst Ueberborst (Berlin 1982) 640; im folgenden zitiert: Größing, Reformen; Hajo Bernett, Der deutsche Sport im Jahre 1933, in: Stadion VII/2 (1982) 272; im folgenden zitiert: Bernett, Der deutsche Sport im Jahre 1933; ein erster systematischer Versuch jetzt bei Jürgen Court, Interdisziplinäre Sportwissenschaft (Frankfurt a.M. u. a. 2000), Teil III; im folgenden zitiert: Court, Interdisziplinäre Sportwissenschaft.

11 Michael Krüger, Einführung in die Geschichte der Leibeserziehung und des Sports, Bd. 2 (Schorndorf 1993) 16; im folgenden zitiert: Krüger, Einführung Bd. 2.

12 Giselber Spitzer, Aktuelle Konzepte zur Zeitgeschichte des Sports unter Berücksichtigung der Diskussion in der Geschichtswissenschaft, in: Sozial- und Zeitgeschichte des Sports 8 (1994) 3, 65; vgl. Hajo Bernett, Neue Aspekte der Zeitgeschichte des Sports, in: Sportwissenschaft 25 (1995) 120.

13 Hubert Dwertmann, Die Rolle Carl Diems im nationalsozialistischen Regime, in: Sozialund Zeitgeschichte des Sports 11 (1997) 2, 11; im folgenden zitiert: Dwertmann, Die Rolle Carl Diems.

${ }^{14}$ Friedrich Schiller, Wallensteins Tod, III 13. 


\section{Zum Idealismusparadigma - historisch-systematische Überlegungen}

Eine detaillierte historisch-systematische Analyse des Idealismusparadigmas besitzt ihren Ausgangspunkt in der programmatischen These, daß

„die Nazi-Romanistik in einen weiteren historischen Rahmen gestellt und nicht nur als ein zeitgeschichtliches Phänomen der deutschen Wirklichkeit von 1933-1945 betrachtet wird, sondern als das Ergebnis eines komplexen Entwicklungsprozesses, der zu Beginn des 19. Jahrhunderts mit der Gründung der Disziplin einsetzte ${ }^{415}$.

Da auch der Anfang der deutschen Turnbewegung 16 bei Friedrich Ludwig Jahn in diesen Zeitraum fällt, kann in einer historischen Perspektive das Idealismusparadigma auf einen gemeinsamen Ursprung von Romanistik und Turnwissenschaft zurückgeführt werden, in dem die Sprache die Funktion eines nationalen Identifikationsmittels einnimmt ${ }^{17}$. Im Zeitalter der Befreiungskriege und Jahns bekanntem "Welschenhaß" 18 ist nicht bloß seine Idee des Turnens eine Objektivation „des sich im Volk ausdrückenden Willens, die französische Fremdherrschaft zu beenden und das Volk [...] in Deutschland zur Herrschaft gelangen zu lassen "19: Fichte, Arndt und eben Jahn nahmen zudem vehement gegen den Französischunterricht Stellung ${ }^{20}$. Und der Gründervater der romanistischen Fachgeschichte, Friedrich Diez (1784-1876), war sogar angeblich zeitlebens stolz darauf, die französische Sprache nur unvollkommen zu beherrschen ${ }^{21}$.

Das ideengeschichtliche Verbindungsglied zwischen den Wirkungen der Französischen Revolution und der Jahrhundertwende ist die „Kultur-Zivilisationsan-

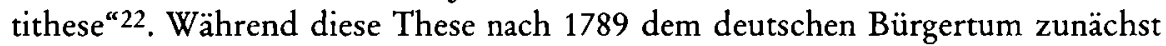

15 Hausmann, Nationale Wissenschaft 1. Teil, 4.

16 Zur Übersicht Hartmut Becker, Turnbewegung, Deutsche, in: Sportwissenschaftliches Lexikon, hrsg. v. Peter Röthig u.a. (Schorndorf 61992) 534-535; ausführlich Michael Krüger, Körperkultur und Nationsbildung. Die Geschichte des Turnens in der Reichsgründungsära eine Detailstudie über die Deutschen (Schorndorf 1996); im folgenden zitiert: Krüger, Körperkultur und Nationsbildung.

17 Vgl. Peter Weingart, Einheit der Wissenschaft - Mythos und Wunder, in: Grenzüberschreitungen in der Wissenschaft, hrsg. v. Peter Weingart (Baden-Baden 1995) 15; im folgenden zitiert: Weingart, Einheit: „Eng verbunden mit nationaler Identität und dem daraus entstehenden Gefühl von Stolz und Überlegenheit als Grenzziehung in der Wissenschaft ist die Sprache. Mit der Sprache, weit mehr noch als mit der Nationalität, verbindet sich kulturelle Identität." Zum Turnen als ,Sprachec vgl. Krüger, Körperkultur und Nationsbildung 422.

18 Friedrich Breier, Ueber Adolf Spieß und sein Turnbuch für Schulen (1852); Nachdruck in: Das gesammte Turnwesen. Ein Lesebuch für deutsche Turner, hrsg. v. Georg Hirth (Leipzig 1865) 531.

${ }^{19}$ Michael Krüger, Einführung in die Geschichte der Leibeserziehung und des Sports, Bd. 1 (Schorndorf 1993) 41.

20 Hausmann, Nationale Wissenschaft 1 . Teil, $18 \mathrm{f}$.

21 Ebd. 16.

22 Christiane Eisenberg, Pferderennen zwischen „Händler-“ und „Heldenkultur“. Verlauf und Dynamik einer englisch-deutschen Kulturbewegung, in: Pionier und Nachzügler? Ver- 
zur Unterstützung des Selbstbewußtseins gegenüber der höfischen Oberschicht diente, trat sie im Gefolge der Napoleonischen Kriege, des Krieges 1870/71 oder der Marokko-Krisen 1905 und 1911 allmählich hinter eine „nationale Antithese [...] zu Frankreich und [...] auch zu England als den Repräsentanten bloßer Zivilisation zurück, der gegenüber Kultur mit Deutschtum mehr und mehr zu einer Begriffssymbiose verschmilzt“, die schließlich die „lebensphilosophisch geprägte Kulturkritik der Jahrhundertwende" 23 durchzieht. Wie in ihrem Gefolge eine „wachsende wechselseitige Radikalisierung der nationaler Konzeptionen zu einer fortschreitenden Systematisierung und scheinbaren Verwissenschaftlichung gegenseitiger Ressentiments" 24 geführt hat, kann an der romanistischen Kulturkunde verdeutlicht werden, die ihren Zweck in der „Erfassung eines Volkscharakters" 25 in der „letzten und allerletzten Literaturgeschichte" 26 hat und in drei Pha$\operatorname{sen}^{27}$ ablief.

In der durch den Ersten Weltkrieg bezeichneten antagonistischen Phase entwirft beispielsweise Hanns Heiß 1916 die Vorstellung einer aggressiven französischen Nation, mit deren Expansionsdrang die Frage der Kriegsschuld zugunsten Deutschlands entschieden ist. Eduard Wechßler verteidigt die Legitimität des deutschen Verteidigungskrieges durch einen phänomenologischen Nachweis unüberbrückbarer mentaler Gegensätze von Deutschen und Franzosen, den er auf die antifranzösischen Argumente der Befreiungskriege stützt ${ }^{28}$. Besonders prägnant kleidet Klemperer diese Phase in einen Tagebucheintrag vom 24. November 1918: „Überhaupt die Franzosen! Wie kann nur ein so niedriges, so gemeines Volk eine solche herrliche Literatur hervorgebracht haben?!"29 Klemperer ist von der Überlegenheit vorgeblich deutscher Tugenden für die Legitimierung des eigenen

gleichende Studien zur Geschichte Großbritanniens und Deutschlands im Zeitalter der Industrialisierung, hrsg. v. Hartmut Berghoff, Dieter Ziegler (Bochum 1995) 235.

${ }^{23} \mathrm{Vgl}$. - im Anschluß an Norbert Elias - Hans-Joachim Lieber, Kulturkritik und Lebensphilosophie. Studien zur Deutschen Philosophie der Jahrhundertwende (Darmstadt 1974) XIV; im folgenden zitiert: Lieber, Kulturkritik; vgl. Stefan Gross, Ernst Robert Curtius und die deutsche Romanistik der zwanziger Jahre. Zum Problem nationaler Images in der Literaturwissenschaft (Bonn 1980) 11; im folgenden zitiert: Gross, Curtius.

24 Gross, Curtius 11.

25 Victor Klemperer, Immer wieder Kulturkunde, in: Neue Jahrbücher für Wissenschaft und Jugendbildung 4 (1928) 273; im folgenden zitiert: Klemperer, Kulturkunde.

26 Victor Klemperer, Alte und neue Neuphilologie, in: Leipziger Neueste Nachrichten vom 10. Oktober 1920, 9; im folgenden zitiert: Klemperer, Neuphilologie.

27 Vgl. Frank-Rutger Hausmann, Die nationalsozialistische Hochschulpolitik und ihre Auswirkungen auf die deutsche Romanistik von 1933 bis 1945, in: Deutsche und österreichische Romanisten als Verfolgte des Nationalsozialismus, hrsg. v. Hans Helmut Christmann, Frank-Rutger Hausmann (Tübingen 1989) 37f.; im folgenden zitiert: Hausmann, Verfolgte; Frank-Rutger Hausmann, „Aus dem Reich der seelischen Hungersnot ${ }^{\text {“ }}$. Briefe und Dokumente zur Fachgeschichte der Romanistik im Dritten Reich (Würzburg 1993) 122; im folgenden zitiert: Hausmann, Reich; Hausmann, Nationale Wissenschaft 1. Teil, $25 \mathrm{ff}$.

${ }^{28}$ Hausmann, Nationale Wissenschaft 1. Teil, $25 \mathrm{f}$.

29 Victor Klemperer, Leben sammeln, nicht fragen wozu und warum. Tagebücher 1918-1932, 2 Bde. (Berlin 1996) 9; im folgenden zitiert: Klemperer, Tagebücher 1918-1932. 
Faches überzeugt, denn „im gewissen Sinn haben die Franzosen in Deutschland die Sprache und Dichtung ihrer Vergangenheit wieder verstehen gelernt ${ }^{a 30}$.

Während in der anschließenden antithetischen Phase unter Berufung auf Dilthey und Spranger die Vertiefung der prinzipiellen Verschiedenheit von Deutschen und Franzosen mit der Betonung der Verflochtenheit von Individuellem und Allgemeinen einhergeht ${ }^{31}$, bedeutet die bumanistische Stufe, die geschichtlich mit dem Vertrag von Locarno 1926 zusammenfällt, eine „Verschiebung der Gewichtung vom Chauvinistischen und Nationalen hin zum Humanitären ${ }^{332}$.

Der Versuch, die eigene Wissenschaft aus der Analyse des Volkscharakters abzuleiten, ist nun auch ein wesentliches Merkmal der frühen Sporttheorie, wie an einer Übertragung der Phasen der romanistischen Kulturkunde auf die beginnende Sportwissenschaft abgelesen werden kann. Die antagonistische Stufe des Weltkrieges kann ebenso durch Diems Rede von "tobenden Völkergegensätzen“ und „verhaßten Feinden“ markiert werden wie durch seine bereits 1914 gewonnene Überzeugung, daß an „Zielsicherheit und Organisationskraft uns vielleicht

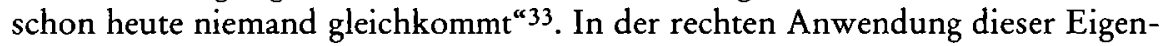
schaften auf die Leibesübungen, deren „ausgeprägte nationale Eigenart die Form der Unübertrefflichkeit ist“, sind sie die Grundlage dafür, daß die „deutsche Rasse unüberwindbar sein wird"34. Daß man diese Phase auch agonale Phase nennen könnte, verdeutlicht Diems Bemerkung, daß es sich „bei den Olympischen Spielen ja [...] gar nicht um die Entscheidung der Frage handelt, ob der Läufer X schneller ist als der Läufer Y, sondern darum, welche Nation imstande ist, [...] ein Bild besonderer körperlicher Rüstigkeit zu geben" ${ }^{\text {"35 }}$.

30 Klemperer, Neuphilologie 9.

31 Hausmann, Verfolgte 37; Hausmann, Nationale Wissenschaft 1. Teil, 26.

32 Hausmann, Verfolgte $37 \mathrm{f}$.

33 Carl Diem, Ausgewählte Schriften, 3 Bde. (St. Augustin 1982) hier: Bd. 2, 87 f.; im folgenden zitiert: Diem, Ausgewählte Schriften. So spricht auch Bruno Barth, Englischer Sport deutsches Turnen (1917), Neudruck in: Der Sport im Kreuzfeuer der Kritik. Kritische Texte aus 100 Jahren deutscher Sportgeschichte, hrsg. v. Hajo Bernett (Schorndorf 1982) 30, in seinem Vergleich von deutschem Turnen und englischem Sport von „scharfen Gegensätzen, [...], die aus der seelischen Natur der beiden Völker geflossen sind. [...] Jedes hat seinem Volke das gegeben, was es brauchte und was in seinen Augen als wertvoll galt: Dieser hat im wesentlichen dazu gedient, eine individualistisch gesinnte, egoistische und aristokratische Herrscherklasse, erobernde Kolonisatoren und rücksichtlos ausbeutende Willensmenschen heranzuzüchten; jenes hat ein wohldiszipliniertes, gründliches, vielseitig gebildetes Volk mit starkem sozialen Gemeinschaftsgefühl heranzuziehen geholfen."

34 Diem, Ausgewählte Schriften, Bd. 2, 119.

35 Ebd. 87. Vgl. auch aus einer Reichtagsrede 1914 von Theodor Lewald, zit. n. Arnd Krüger, „Dann veranstalten wir eben rein deutsche Olympische Spiele!“ Die Olympischen Spiele von 1936 als deutsches Nationalfest, in: Schwimmsport und Sportgeschichte, hrsg. v. Helmut Breuer, Roland Naul (St. Augustin 1994); im folgenden zitiert: Krüger, Nationalfest 134: „Es ist ein Nachteil für Deutschland gewesen, und es hat mit dazu beigetragen, daß Deutschland bei den früheren Spielen nicht so besonders günstig abgeschnitten hat, daß immer das einladende Land im wesentlichen seine Spielregeln zugrunde legt. [...] Wenn wir die Gastgeber sind, die einladen, dann können wir natürlich einen ganz anderen Einfluß darauf ausüben, daß diese Spielregeln in unserem Sinne abgefaßt werden. ${ }^{*}$ 
In der antithetischen Phase betont zwar Diem 1920, daß die Antwort auf die rechte Weise der Leibesübungen nicht der "nationalen Eigenart", sondern der "menschenfreundlichen Liebe“ 36 entspringe. Er geht aber nicht so weit, das noch 1919 als „Erzfeind“ ${ }^{37}$ bezeichnete Frankreich als Austragungsort der Olympischen Spiele 1924 für die deutschen Teilnehmer zu akzeptieren, da man aus den Olympischen Spielen

„im Jahre 1920 in Antwerpen eine Entente-Olympiade unter Ausschluß der Mittelmächte machte und diesen Zustand offenbar zu verewigen gesucht hat, indem man zum Kampfplatz des Jahres 1924 ausgerechnet Paris wählte. Die Deutschen sind also gar nicht in der Lage, an den Internationalen Spielen teilzunehmen, und es ist sehr fraglich, ob sie wieder den Wunsch dazu in späterer Zeit überhaupt haben werden“"38.

Für sein Ideal einer „deutschen Volkseinheit“ haucht Diem vielmehr im Jahre 1921 dem alten Plan „deutscher Kampfspiele“"39 neues Leben ein, und seine bis ins letzte Detail ausgetüftelten organisatorischen Vorschläge sind Spiegel jenes Glaubens an die deutschen Wesenzüge ,Zielsicherheit' und ,Organisationskraft'. Und auch die humanistische Phase läßt sich bei Diem nachweisen, wenn er 1931 die Notwendigkeit einer Besinnung auf die "großen Erzieher aller Zeiten“ und insonderheit auf die „Blütezeit der griechischen Kultur" 40 betont.

Eine ausgezeichnete systematische Funktion für das Idealismusparadigma der Weimarer Zeit nimmt in diesem historischen Ablauf der Erste Weltkrieg ein ${ }^{41}$. Diems Überzeugung, daß der „Krieg auch unser Gebiet wie mit einer Fackel erhellt hat ${ }^{\text {"42 }}$, entspricht Klemperers programmatischer Überzeugung, mit dem "Ende des Krieges“ sei ein notwendiger Einschnitt in der Erörterung von „Begriff und Möglichkeit, Gefahr und Notwendigkeit der Kulturkunde" 43 gekommen. Da das deutsche Volk "vor der Riesenaufgabe steht, seine wirtschaftliche Weltgeltung nach dem Kriege wieder so bald als möglich im alten Verhältnis erstehen zu las-

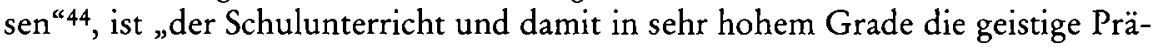
gung der kommenden Generation davon abhängig, was man unter Kulturkunde versteht" ${ }^{45}$. Wenn Diem also vom Sport fordert, er solle „lebenstüchtig“46 machen, und Klemperer in der Romanistik seinen Idealismus als Gegenposition zu einer positivistischen Richtung konzipiert, deren Beharren auf „absoluter Begriffsreinheit " in letzter Hinsicht das "Leben " 47 verfehle, ist diese Betonung der

36 Diem, Ausgewählte Schriften, Bd. 1, 38.

37 Diem, Ausgewählte Schriften, Bd. 2, 137.

38 Ebd. 158.

39 Ebd. $157 \mathrm{f}$.

40 Ebd. 206, 222.

$41 \mathrm{Vgl}$. Hausmann, Nationale Wissenschaft 1. Teil, $6 \mathrm{f}$.

42 Diem, Ausgewählte Schriften, Bd. 2, 113.

43 Klemperer, Kulturkunde 264.

44 Diem, Ausgewählte Schriften, Bd. 2, 125.

45 Klemperer, Kulturkunde 264.

46 Diem, Ausgewählte Schriften, Bd. 2, 131.

47 Klemperer, Neuphilologie 9. 
„am lebendigen Fleische gewonnenen Erfahrungen“48 das gemeinsame wissenschaftstheoretische Gerüst von Diem und Klemperer: Ihr lebensphilosophischer Ansatz besteht vor der Folie der Kriegserfahrung in einer unaufhebbaren Verbindung von Theorie und Praxis, von Wissenschaft und Erziehung.

Betrachten wir zunächst die wissenschaftstheoretische Seite in Diems Lesart „Es ist nicht nur, wie Schiller sagt, ,Der Geist, der sich den Körper baut', sondern auch ,der Körper, der sich den Geist baut " ${ }^{\text {" } 49}$, und Klemperers Bestimmung der Kulturkunde, „festzustellen, was ist, wie es geworden ist, wie sich das Einzelne ins Ganze einordnet, wie sich der Geist den Körper baut " ${ }^{50}$. Während für Klemperer eine an Schillers Idealismus ausgerichtete Sprachwissenschaft die Funktion besitzt, "auch so mechanischen Vorgängen wie dem Lautwandel geistig befriedigende Deutung "51 zu geben, setzt Diem seine ursprünglich gegen das Schwedische Turnen gezielte gleichermaßen antipositivistische und antimaterialistische Tradition 52 als Waffe gegen die "traurige Orthopädie“ des Deutschen Turnens ein:

„Nicht geistlose und schematische Körperübung ist das Ziel der sportlichen Bewegung [...]. Das sportliche Ideal heißt , persönliche Höchstleistung [...]. Sport ist idealistisch oder er ist kein Sport. Daher erfüllt der Sport die entscheidende Aufgabe einer Erziehung im Geiste des Idealismus, er zeigt der Jugend, daß das Ideale das einzig reale, das einzig kraftgebende, das einzig unzerstörbare Gut im Dasein ist [...]. Das Gift kommt vom Materialismus. " 53

Sowohl Klemperers Kritik an Groebers Französischer Literatur mit ihrem „nackten und unanfechtbaren Tatsachenmaterial“ 54 als auch Diems Forderung, „das Turnen muß den Begriff einer technischen Bewegungskunst verlieren ${ }^{\text {“55, markie- }}$ ren eine „in der Bildung begriffene neue Wissenschaft ${ }^{\text {" }} 5$, deren Notwendigkeit pädagogischen Charakter trägt. Diems Einwürfe gegen geisteswissenschaftliche Schulstunden ${ }^{57}$ bedeuten vor dem Klemperer und Diem gemeinsamen idealistischen Menschenbild keinen Gegensatz in ihrem Ziel einer „Erziehung des ganzen

48 Diem, Ausgewählte Schriften, Bd. 2, 67.

49 Ebd. 200.

50 Zit. n. Bock, Zur Stellung Victor Klemperers 104; das Zitat stammt aus dem Jahre 1924. Ein späteres Zitat aus dem Jahre 1945 bei Victor Klemperer, So sitze ich denn zwischen allen Stühlen. Tagebücher 1945-1949 (Berlin 1999) 98; im folgenden zitiert: Klemperer, Tagebücher 1945-1949.

51 Victor Klemperer, Romanische Sonderart (München 1926) 395; im folgenden zitiert: Klemperer, Sonderart. Auch Klemperers Konkurrent und Kollege Ernst Robert Curtius hatte ein „eher gebrochenes Verhältnis zur Phonetik “, denn er schrieb in einer Universitätsgeschichte: „Der Einbruch der Phonetik in den philologischen Lehrbetrieb läßt sich aus dem Antrag auf Beschaffung eines menschlichen Kehlkopfes (Knorpelmodell mit spannbaren Stimmbändern) erkennen, der am 13. Juni 1902 genehmigt wird“ (zit. n. Hausmann, Reich 68).

$52 \mathrm{Vgl}$. Court, Ideengeschichte 142, $152 \mathrm{ff}$.

53 Diem, Ausgewählte Schriften, Bd. 1, 38, 46, 156, 203.

${ }^{54}$ Klemperer, Sonderart 392.

55 Diem, Ausgewählte Schriften, Bd. 1, 51.

56 Klemperer, Sonderart 390.

57 Vgl. Diem, Ausgewählte Schriften, Bd. 2, 200: „Wegen der Abtretung von 4 geisteswissenschaftlichen Stunden zugunsten des Turnens braucht ein Rückgang der deutschen Geisteshöhe nicht befürchtet zu werden." 
Menschen"58, sondern bloß eine Warnung vor den Übertreibungen des „einseitigen Intellektualismus“59 bzw. der „Lautschieberei“60. Klemperers Kampf gegen die Erstarrung des Positivismus und Diems Invektive gegen den Schematismus des Turnens sind daher zwei - vom Blickpunkt des jeweiligen wissenschaftlichen Gegenstands abhängige - Seiten derselben Medaille des "wahrhaft Lebenden“61.

Den Schlüssel zum Verständnis der Beziehung zwischen Kontinuitätsfrage und Idealismusparadigma erhalten wir, wenn wir einen näheren Blick auf die Argumente werfen, mit denen Diem und Klemperer ihren jeweiligen Begriff der Kultur untermauern. Klemperer etwa begründet den Vorrang der französischen vor der spanischen Sprache ${ }^{62}$ mit Begriffen wie dem „Kern der Wesensart“, der „Blutnähe" oder dem "Seelenpendel“63, und Diems Ziel einer "weit ausschauenden planmäßigen Volkshygiene" hat einen "Gebärprozeß zur Voraussetzung“, der aus einem „Zustand der Gärung “64 erwächst. Die Problematik einer solchen für die Kultur-Zivilisations-Antithese markanten Übertragung von Kategorien des Lebens auf das der Kultur ${ }^{65}$ hellt sogleich auf, wenn wir sie an die lebensphilosophische Identifizierung des „Lebendigen“" mit dem „Ewigen“66 und die Übertragungsmechanismen durch zwei ihrer wichtigsten Vertreter, Wilhelm Dilthey und Eduard Spranger, halten. Diems aus einem theoretischen Antipositivismus hervorgegangene Lebensphilosophie wirkte über Vossler ${ }^{67}$ auf Klemperer und über den Dilthey-Schüler Spranger auf Carl Diem ${ }^{68}$. Sprangers antipositivistische Rede von „Urkategorien“ und "Urphänomenen"69 oder Diltheys Bezeichnung der

58 Diem, Ausgewählte Schriften, Bd. 2, 200.

59 Ebd. 151; vgl. ebd. 125: „Dem Geiste, was des Geistes ist, aber - mag es auch in dieser Fassung als revolutionierendes Wort gescholten werden -, aber: auch dem Körper, was des Körpers ist." Dies bedeutet daher keinen „Anti-Intellektualismus“, der üblicherweise als Bindeglied zur NS-Ideologie angesehen wird (vgl. Joch, Sport 703; Bernett, Der deutsche Sport im Jahre 1933 272).

60 Klemperer, Neuphilologie 9.

61 Ebd. 9.

62 Klemperer, Sonderart 410: „Und so darf u. muß man wohl eine Rangordnung unter den großen romanischen Literaturen aufstellen: die französische steht an erster Stelle, an zweiter die italienische u. die spanische an dritter."

${ }^{63}$ Victor Klemperer, Einleitung, in: Victor Klemperer, Helmuth Hatzfeld, Fritz Neubert, Die romanischen Literaturen von der Renaissance bis zur französischen Revolution (Potsdam 1928) 14.

64 Diem, Ausgewählte Schriften, Bd. 2, 35, 39, 154.

65 Vgl. zur Bedeutung Nietzsches für dieses biologistische Begründungsschema Court, Interdisziplinäre Sportwissenschaft 192-196.

${ }^{66} \mathrm{Vgl}$. Klemperers (Victor Klemperer, Romanische Sonderart $397 \mathrm{f}$.) Lob des Anglisten Max Deutschbein, für den eine phänomenologische Orientierung am „Lebendigen, Dauernden, Ewigen der geistigen Gebilde [...] schöpferische Lebensschule" sei.

67 Hausmann, Nationale Wissenschaft 1. Teil, 14.

$68 \mathrm{Vgl}$. Helmut E. Lück, Zu Eduard Spranger und dessen Verhältnis zu Carl Diem und der Leibeserziehung, in: Sportonomics 3 (1997) 1, 39; im übrigen war Spranger auch ein gewichtiger Kronzeuge der idealistischen Romanistik (vgl. Hausmann, Verfolgte 37).

69 Zit. n. Bernd Wirkus, Deutsche Sozialphilosophie in der ersten Hälfte des 20. Jahrhunderts (Darmstadt 1996) 134. 
Ideen der Französischen Revolution als „Unwahrheit“" Verbindung von Ahistorizität und Biologismus zu einem das Idealismusparadigma durchziehenden Widerspruch zwischen vorgeblich rationaler und faktisch irrationaler Begründung von Wissenschaft. Die wirkungsgeschichtliche Problematik des aus ihm resultierenden Dezisionismus besteht aber

„nicht so sehr in der Abstraktheit und Inhaltsleere seines Entscheidungsbegriffes, als vielmehr darin, daß der beschworene Aktivismus der Entscheidung, weil dieser das inhaltliche Sich-Einlassen mit der konkreten Wirklichkeit [...] sich versagt, von ebendieser Wirklichkeit selber ereilt wird, als Begleitphänomen an gesellschaftliche Inhalte und Bewegungen sich engagiert, die - wenn sie nur geschichtliche Entschiedenheit propagieren - als seine okkasionelle Bewährung und Vollendung fungieren können " 71 .

In dieser dem Dezisionismus inhärenten Vermischung der Genese und der Geltung wissenschaftlicher Erkenntnis rückt daher die Bedeutung von Klemperers und Diems „Primärsozialisation “72 im Wilhelminismus und im Ersten Weltkrieg in den Mittelpunkt. Dieses subjektive Implikat des Idealismusparadigmas besagt, daß Klemperer, als er - anders als der ,Arier' Diem - als Jude eben nicht politisch gewünschte Rollenfunktionen einnehmen kann und in eine Außenseiterposition gedrängt wird ${ }^{73}$, verstärkt skeptisch-kritische Positionen einnimmt.

Man muß hier ergänzen, daß außer fast identischen Lebensdaten (Klemperer lebte von 1881 bis 1960 und Diem von 1882 bis 1962) bis 1933 weitere bis ins Detail gehende biographische Übereinstimmungen in Diems und Klemperers Sozialisation als Angehörige der „Frontkämpfer-Generation"74 festgehalten werden können. Beide besuchten in Berlin zunächst das Königlich Französische Gymnasium und wechselten dann auf das Friedrich-Werdersche Gymnasium, das Klemperer 1897 und Diem 1900 als "Einjährige" verließen, um anschließend eine Kaufmannslehre zu beginnen ${ }^{75}$; beide immatrikulierten sich 1904 an der Universität Berlin, hörten in den gleichen Fächern ${ }^{76}$ und bei den gleichen Professoren ${ }^{77}$. Beide

70 Zit. n. Lieber, Kulturkritik $113 \mathrm{f}$.

71 Lieber, Kulturkritik 117.

72 Dwertmann, Die Rolle Carl Diems 11.

73 Vgl. zu diesem methodischen Ansatz Dwertmann, Die Rolle Carl Diems 9: „Die Gewichtigkeit einer solchen Rollendeutung wird ersichtlich, wenn der Ausschluß aus dem nationalsozialistischen Akzeptanzspielraum von [...] Kommunisten, Sozialdemokraten, Juden, Emigranten usw. 1933 im Vergleich zur Diemschen Rolle herangezogen wird.“

${ }_{74}$ Hans Joachim Teichler, Der Weg Carl Diems vom DRA-Generalsekretär zum kommissarischen Führer des Gaues Ausland im NSRL, in: Sozial- und Zeitgeschichte des Sports 1 (1987) 1, 46; im folgenden zitiert nach: Teichler, Der Weg Carl Diems; ausführlich zum Thema Heinrich August Winkler, Weimar 1918-1933. Die Geschichte der ersten deutschen Demokratie (München 1998) 607 ff.; im folgenden zitiert: Winkler, Weimar.

75 Carl-Diem-Institut (Hrsg.), Dokumente zum Aufbau des deutschen Sports. Das Wirken von Carl Diem (1882-1962) (St. Augustin 1984) 5 f.; im folgenden zitiert: Carl-Diem-Institut, Dokumente; Victor Klemperer, Curriculum Vitae. Jugend um 1900, 2 Bde. (Berlin 1989) $133 \mathrm{ff} . ;$ im folgenden zitiert: Klemperer, Curriculum.

${ }^{76}$ Carl-Diem-Institut, Dokumente 8; Klemperer, Curriculum Bd. 1, 304, $352 \mathrm{ff}$.

77 Dies waren neuere deutsche Literatur bei Erich Schmidt und Ludwig Geiger sowie Philosophie bei Max Dessoir; vgl. Carl Diem, Ein Leben für den Sport. Erinnerungen aus dem 
waren journalistisch tätig - Diem als Sport- und Klemperer als Feuilletonredakteur ${ }^{78}$-; Diem und Klemperer waren freiwillige Teilnehmer des Weltkrieges, in dem sie - so Klemperer - Deutschland ,im allerreinsten Recht “79 wähnten. Ihr Verhältnis zur Weimarer Republik ist dasjenige, was die Rede von einer „Republik ohne Republikaner" 80 ausdrückt; Klemperer empfiehlt im Mai 1924 mit der nationalliberalen DVP eine Partei zur Reichstagswahl, deren Mitglied Diem ist ${ }^{81}$, Klemperer ${ }^{82}$ und Diem ${ }^{83}$ stehen jedoch prinzipiell dem Parteiensystem mit Distanz gegenüber und votieren im April 1932 für Hindenburg, für den Diem öffentlich wirbt ${ }^{84}$.

Unsere These besagt also, daß die Vertreter der idealistischen Lebensphilosophie das in das Idealismusparadigma der Weimarer Republik eingeflossene nationalkonservative Gedankengut in den Nationalsozialismus wissenschaftlich integrieren konnten, insofern ihr biographischer Hintergrund nicht diesem Diskriminierungskriterium der jüdischen Herkunft unterlag. Klemperers Tragik als Jude besteht darin, daß seine Erklärung sprachlicher Phänome durch die nationalsozialistische Sprachwissenschaft instrumentalisiert werden konnte, ohne daß er sich dagegen öffentlich zu wehren vermochte, eben weil er von dieser Umfunktionalisierung selbst, in seiner ganzen Existenz, getroffen wurde. Deshalb mußte er schweigend solche Sätze wie den Glässers aus dem Jahre 1939 erdulden: Das

„rassische Herrentum und seine schöpferische Leistung stellt den Geist dar, der sich den Körper des rassisch andersartig durchschossenen Gemeinschaftsgebildes baut. Dieser Geist aber ist der Geist des das Leben spendenden [...] Blutes, an das Blut ist der Geist gebunden" ${ }^{485}$.

Nachlaß (Ratingen u.a. o. J.) 29; im folgenden zitiert: Diem, Ein Leben; Klemperer, Curriculum Bd. 1, $354 \mathrm{ff} .$, 359. Haben sich vielleicht Diem und Klemperer - zumindest vom Sehen her - gekannt?

78 Carl-Diem-Institut, Dokumente 9; Klemperer, Curriculum Bd. 1, $408 \mathrm{ff}$.

79 Klemperer, Curriculum Bd. 1, 179. Wenn Diems Offizierslaufbahn nach Hajo Bernett, Carl Diem, in: Sportwissenschaftliches Lexikon, hrsg. v. Peter Röthig (Schorndorf 61992) 118 , auf „innerer Zustimmung“ beruhte, ist dies mißverständlich. Diem, Ein Leben 94, 96, ist zwar "freiwillig ins Feld gezogen“, konnte jedoch die „jubelnde Begeisterung “ seiner Freunde "nicht teilen“. Diem nennt als Motiv der freiwilligen Meldung lediglich "Pflicht". 80 Frank Golczewski, Kölner Universitätslehrer und der Nationalsozialismus. Personengeschichtliche Ansätze (Köln 1988) 43.

81 Klemperer, Tagebücher 1918-1932, Bd. 1, 808; Teicbler, Die Rolle Carl Diems 70, Anm. 65.

82 Vgl. Klemperer, Tagebücher 1918-1932, Bd. 2, 434: „Die Reichstagswahl am 20/5 [1928. J.C.] machte geringen Eindruck auf mich. Es ändert sich ja so wenig an den Verhältnissen. [...] Übrigens gehört mein Herz keiner Partei mehr gänzlich.“

83 Teichler belegt Diems Distanz anhand eines Satzes, den dieser anläßlich der Präsidentenwahl 1932 in der Vossischen Zeitung schrieb: „Wir wollen glücklich sein, wenn nicht auch noch das Amt des Reichspräsidenten parteipolitisch gebunden ist " (zit. n. Teichler, Die Rolle Carl Diems 70, Anm. 65).

84 Vgl. Teichler, Die Rolle Carl Diems 70, Anm. 65; Klemperer, Tagebücher 1918-1932, Bd. 2, 744.

85 Zit. n. Frank-Rutger Hausmann, Auch eine nationale Wissenschaft? Die deutsche Roma- 
Diem hingegen konnte den Nationalsozialismus als Chance der Verwirklichung seiner eigenen Vorstellungen auffassen. Wenn wir Hitlers Eingangszitat mit seiner Warnung vor bloßer Geistesbildung und der Forderung nach einem zweistündigen Pflichtsport mit Diems schon 1912 verlangten täglichen Turnstunde und seiner Abwehr des, einseitigen Intellektualismus' vergleichen, dann vermögen wir nur aufgrund der schärferen Tonart Hitlers zu unterscheiden, ob es von Diem oder Hitler stammte.

„Ja, kann man denn aber - so fragen die Schwachnervigen - dem jungen Mann nun jede Freiheit nehmen? Warum denn nicht, sage ich, wenn es nur zu seinem Besten ist. Die erste Forderung müßte sein, die Jugend zu regelmäßigen Körperübungen heranzuziehen. Erst dann, nicht umgekehrt, kommt der geistige Unterricht. Will man dem Jungen freie Tage absolut lassen - gut, dann streiche man am geistigen Unterricht; wer den anderen streicht, schlägt sein Volk tot. [...] Auch das 20. Jahrhundert hat den größten Unsinn der Weltgeschichte, die klingende Phase von der Freiheit (in der peinlichen Gesellschaft von Gleichheit und Brüderlichkeit) noch nicht überwinden können. [...] Was meintet ihr, würde das ein Geschlecht von Führern geben, wenn unsere Studenten jeden Tag zwei Stunden vormittags oder nachmittags einen frei gewählten Sport pflichtmäßig betrieben!" ${ }^{86}$

Vor diesem Hintergrund bedeuten die folgenden Kapitel auch eine genaue Prüfung dieser prima facie so großen Übereinstimmung zwischen den zitierten Äußerungen Diems und Hitlers: Auf welche Traditionen konnte sich Diem unter den Bedingungen der ,Gleichschaltung berufen, und mit welchen mußte er brechen ${ }^{87}$ ?

Da das Thema dieses Beitrages sich auf die Sportwissenschaft an deutschen Hochschulen beschränkt, geht es zunächst um die Analyse der Hochschulsportordnung von 1934 und dann um die der ,Reichsakademie für Leibesübungen“. Ein Fazit wird anhand der wissenschaftlichen Bedeutung der Olympischen Spiele von 1936 gezogen.

\section{Sportwissenschaft im Nationalsozialismus}

\subsection{Zur Hochschulsportordnung von 1934}

Victor Klemperer notiert in seinem Tagebuch vom 11. Februar 1937 den Brief eines befreundeten Kollegen:

„Wengler erzählte, er sei für einen Kurs in ein ,Lager ‘ bei Königs Wusterhausen geschickt worden. Studienräte zwischen vierzig und fünfzig Jahren. Man schlief zu sechsen, trug Uniform, machte in Erdarbeit und Sport, bekam bildende Vorträge. Ein Studiendirektor sprach über den Charakter der Franzosen; sie seien ähnlich wie die Juden, sie hätten keine Liebe zum Tier." 88

nistik unter dem Nationalsozialismus. 2. Teil, in: Romanistische Zeitschrift für Literaturgeschichte 22 (1998) $268 \mathrm{ff}$.

${ }_{86}$ Diem, Ausgewählte Schriften, Bd. 2, $60 \mathrm{f}$.

87 Auch Teicbler, Die Rolle Carl Diems 56, betont die methodische Notwendigkeit eines solchen Vergleichs.

88 Victor Klemperer, Ich will Zeugnis ablegen bis zum letzten. Tagebücher 1933-1941 (Berlin 
Klemperer verdichtet hier in wenigen Zeilen ein Programm, an dem exemplarisch die Ziele der nationalsozialistischen Machtpolitik verdeutlicht werden können: die in ihrem ersten Teil mit Erlaß vom 30. Oktober 1934 in Kraft getretene Hochschulsportordnung (HSO). Sie war als erste größere Maßnahme des neuen Amtes $\mathrm{K}$ für körperliche Erziehung im Reichsministerium für Wissenschaft, Erziehung und Volksbildung unter Bernhard Rust (einem früheren Studienrat für Deutsch, Philosophie, Latein und Sport ${ }^{89}$ ) das erste geschlossene, für einen Teilbereich gültige Konzept der Nationalsozialisten und diente der Organisation und inhaltlichen Gestaltung der Leibesübungen unter der Prämisse weltanschaulicher Erziehung. Die HSO dokumentiert nicht nur den Prozeß der Gleichschaltung im Bereich der Kultur, sondern auch das Bemühen, unterschiedliche Konzeptionen von Leibesesübungen zentral zu lenken und klare Kompetenzen zu schaffen. Indem der Chef des Amtes K, Carl Krümmel, für alle Studenten eine dreisemestrige Pflichtsportausbildung verordnete, die er in seiner militärischen Sprache,Grundausbildung' nannte, beschränkte er gleichzeitig die Macht der SA, deren PflichtSportabzeichen nun in die HSO integriert wurde 90 .

Die Eingliederung der HSO in unsere Leitfrage nach Kontinuität und Diskontinuität verlangt zwei heterogene Entwicklungslinien ihrer akademischen Vorgängerinstitutionen zu betrachten. Erstens hat der Gedanke eines studentischen Sports eine lange Tradition, die bis zu ersten Überlegungen des Zentralausschusses für Volks- und Jugendspiele aus dem Jahre 1901 reicht ${ }^{91}$. Diems oben zitierte Idee studentischen Pflichtsports ${ }^{92}$ fand nach dem Versailler Vertrag und der Abschaffung der Wehrpflicht große Zustimmung sowohl im DRA (Deutscher Reichsausscbuß für Leibesübungen) mit seinem Vorsitzenden Theodor Lewald und seinem Generalsekretär Diem wie in den mehrheitlich rechtsnationalen studentischen Kreisen, weil man von ihm einen „Ersatz für die verlorene Gesundheitsquelle des

1995) 337; im folgenden zitiert: Klemperer, Tagebücher 1933-1941. Vgl. Victor Klemperer, LTI. Notizbuch eines Philologen (Berlin 1947); im folgenden zitiert: Klemperer, LTI 41: "Plötzlicher Ukas, sehr einschneidend in den Lehrplan der Hochschule: der Dienstagnachmittag ist freizuhalten von Vorlesungen, die Studenten in ihrer Gesamtheit werden in diesen Stunden zu Wehrsportübungen herangezogen" (Tagebuch vom 29. Oktober 1933).

$89 \mathrm{Vgl}$. Frank-Rutger Hausmann, „Deutsche Geisteswissenschaft" im Zweiten Weltkrieg. Die "Aktion Ritterbusch“ (1940-1945) (Dresden, München 1998) 39; im folgenden zitiert: Hausmann, Ritterbusch

90 Bernett, Der deutsche Sport im Jahre 1933 262ff.; Wolfgang Buss, Lorenz Peiffer, 50 Jahre Hochschulsportforschung, in: Sportwissenschaft 16 (1986) $38 \mathrm{ff}$; im folgenden zitiert: Buss, Peiffer, Hochschulsportforschung; zu Carl Krümmel vgl. Horst Ueberborst, Carl Krümmel und die nationalsozialistische Leibeserziehung (Berlin u.a. 1976); im folgenden zitiert: Ueberhorst, Krümmel; Krüger, Einführung Bd. $2141 \mathrm{f}$.

91 Ursula Jost-Hutflesz, Sport und Sportlehrerausbildung an der Universität Heidelberg von 1844-1950, in: Zeitschrift für Sozial- und Zeitgeschichte des Sports 1 (1987) 2, $81 \mathrm{f}$.

92 Diese von Diem 1912 gegen die "Schwachnervigen“ (siehe oben) gerichtete Idee folgt dem allgemeinen Grundzug des Kaiserreichs als „Zeitalter der Nervosität"; vgl.Joachim Radkau, Das Zeitalter der Nervosität. Deutschland zwischen Bismarck und Hitler (München, Wien 1998). 
ehemaligen Heeres ${ }^{493}$ und ein Mittel gegen den demokratischen Weimarer Verfassungsstaat erblickte. Die 1920 auf dem Göttinger Studententag beschlossenen „Leitsätze für die körperliche Erziehung an den Hochschulen“ halten vor diesem Hintergrund im $\$ 3$ fest: "Jeder Studierende ist verpflichtet, während seiner Studienzeit Leibesübungen zu treiben. ${ }^{\text {"94 }}$ Die Berechtigung, gegen eine „einseitige Vergeistigung" das Ideal der "Wehrhaftmachung "95 zu setzen, weist zudem auf den Tenor der zeitgenössischen Rezeption Friedrich Ludwig Jahns ${ }^{96}$.

Die großen Summen, die zum Beispiel der preußische Innenminister zu Beginn der 20er Jahre der Universität Köln „, für die Beteiligung an sportlichen und turnerischen Wettkämpfen innerhalb und außerhalb des besetzten Gebietes“" zur Verfügung stellte, zeigt, daß die Politik sehr rasch die propagandistische Rolle des Sports im Kulturkampf mit Frankreich erkannt hatte. Zuständig für den Hochschulsport war durch Erlaß vom 22. Dezember 1920 der Akademische Ausschuß für Leibesübungen (AAfL), in Köln übrigens mit dem Mitglied Etienne Lorck.

93 Theodor Lewald, zit. n. Buss, Peiffer, Hochschulsportforschung 41. Zum DRA siehe Erich Beyer, Sport in der Weimarer Republik, in: Leibesübungen und Sport in Deutschland vom Ersten Weltkrieg bis zur Gegenwart, hrsg. v. Horst Ueberhorst (Berlin 1982) 659; im folgenden zitiert: Beyer, Sport.

${ }_{94}$ Buss, Peiffer, Hochschulsportforschung 41; zu dieser Ersatzfunktion der Leibesübungen vgl. Beyer, Sport 663, 666, 689. Diese Tradition betont auch Klemperer, LTI 41, im Tagebuch vom 29. Oktober 1933 anläßlich einer Wehrsportübung der Hochschule: „Allgemeine Wehrpflicht ist durch den Versailler Vertrag verboten; Sport ist erlaubt - wir tun offiziell nichts Unerlaubtes, aber ein bißchen tun wir es doch, und machen eine kleine Drohung daraus, wir deuten immerhin die Faust an, die sich - vorläufig noch - in der Tasche ballt". Diese Stelle ergänzt Zitate und Kommentar bei Jürgen Court, Victor Klemperer als Zeitzeuge des Sports, in: Stadion XXIII (1997) 120.

95 Vgl. Friedrich Ludwig Jahn, Ernst Eiselen, Die Deutsche Turnkunst (Berlin 1816, Neudruck Stuttgart 1967) 209: „Die Turnkunst soll die verloren gegangene Gleichmäßigkeit der menschlichen Bildung wieder herstellen, der bloß einseitigen Vergeistigung die wahre Lebhaftigkeit zuordnen."

96 Vgl. die Zusammenfassung von Reinhard K. Sprenger, Zur Jahnrezeption in der Weimarer Republik, in: Stadion VIII/IX (1982/1983) 170f.: "Jahnbild und -urteil in der Weimarer Zeit wurden geprägt von dem Bewußtsein, daß 100 Jahre zuvor, also zu Jahns Lebzeiten, eine offensichtlich vergleichbare politische Katastrophe mit dem Untergang des Reiches 1806 vorausgegangen war. Die Kriegs- und Krisenzeiten 1792-1815 und die scheinbare Analogie zwischen dem Deutschland nach 1806 und der Republik nach der Niederlage 1918 als jeweils Situationen nationaler Demütigung und Entindividualisierung wurden entsprechend vergegenwärtigt und mit aktivistischer Intention nutzbar gemacht. Diese Voraussetzung ließ für einen nicht unbedeutenden Teil der politisch-historischen Publizistik den Jahnschen, Freiheit und nationale Größe vereinigenden Kampfgeist zum entscheidenden Ferment im anzustrebenden Prozeß nationaler Wiederaufrichtung werden. Entsprechend wurden die Rezeptionsakzente im Gegensatz zur Kaiserzeit jetzt auf das politisch-agitatorische, das ,bewegende' Moment des Traditionskomplexes Jahn verschoben, nunmehr das Ideal politischer Selbstbestimmung als Katalysator des Nationalgefühls instrumentalisiert." Dazu paßt ein Zitat von Diem, Ausgewählte Schriften, Bd. 2, 137, aus dem Jahre 1919: „Unseres Volksheeres als Turnlehrstätte beraubt, müssen wir in der freien Turn- und Sportbewegung einen Ersatz schaffen, was uns nur gelingt, wenn die Nationalversammlung schon bei der Verfassungsarbeit die Grundlage dafür legt. Möge doch in ihr ein neuer Friedrich Ludwig Jahn entstehen, der erkennt, daß hier die Wurzeln späterer Kraft einzusetzen und zu heben sind!“ 
Die Organisation des per Erlaß des Preußischen Kultusministers vom 24. 3.1925 verordneten zweisemestrigen Pflichtsports für alle Studenten war dann Aufgabe der per Erlaß vom 30. September 1925 gegründeten Institute für Leibeserziebung (IfL) ${ }^{97}$.

An dieser Stelle sind wir an einer zweiten Entwicklungslinie der IfL angelangt. In einem Erlaß des Preußischen Kultusministers vom 3. März 1926 wurde neben dem Gebiet des Verwaltungswesens und dem des Unterrichtswesens, in das die gesamte Turnlehrerausbildung und der Pflichtsport fielen, nun explizit das Aufgabengebiet der Forschungstätigkeit genannt. Das hier zum Ausdruck kommende Interesse an der Wissenschaftlichkeit des Sports zeugt nicht nur von dem Wunsch, dem Mangel an geeigneten akademischen Turnlehrern abzuhelfen ${ }^{98}$, sondern ist auch Ausdruck der reformpädagogischen Überzeugung von der Bedeutung der körperlichen Erziehung für die Gesamterziehung, der ein verändertes Konzept der Lehre und Forschung des Sports zu entsprechen habe ${ }^{99}$. Vor allem seine medizinischen und philosophischen Aspekte sollten hier in enger Zusammenarbeit mit den jeweiligen traditionellen Fakultäten untersucht werden, was jedoch auf starke Widerstände der meisten preußischen Universitäten stieß, die ,fehlende Wissenschaftlichkeit' befürchteten. Gleichwohl setzten sich die IfL mit ihrer Position durch, und mit Erlaß vom 1. August 1929 konnte ab dem Sommersemester 1930 in Preußen das Fach Leibeserziehung als Haupt- oder Nebenfach in einem achtsemestrigen wissenschaftlichen Studium belegt werden ${ }^{100}$. Ferner erschienen in den 20er Jahren die ersten Schriftenreihen für Leibesübungen und Sportwissenschaft, und im November 1928 konnten sich Vertreter von Turnen, Sport und Leibeserziehung auf die Gründung einer Wissenschaftlichen Gesellschaft für körperliche Erziehung einigen. Sie bekannte sich ausdrücklich zur Verbindung natur- und geisteswissenschaftlicher Verfahren ${ }^{101}$.

Was die nationalsozialistische HSO von 1934 für unser Thema der Kontinuität und Diskontinuität der Sportwissenschaft bedeutet, läßt sich exemplarisch an ei-

97 Vgl. Franz Nitsch, Die Geschichte des Kölner Hochschulsports. Von den Anfängen bis in die Zeit des Nationalsozialismus, in: Sozial- und Zeitgeschichte des Sports 1 (1987) 2, $31 \mathrm{f}$.; zu Verbindungslinien zwischen Schul- und Hochschulsport vgl. ferner Beyer, Sport $665 \mathrm{ff}$. Jene Funktion Lorcks wird nicht erwähnt bei Bernhard König, Etienne (Stefan) Lorck und die Anfänge der Romanischen Philologie in Köln. Handelshochschule (1901-1919) und Universität (1919-1930), in: Lingua et traditio. Geschichte der Sprachwissenschaft und der neueren Philologien. Festschrift für Hans Helmut Christmann zum 65. Geburtstag, hrsg. v. Richard Baum u. a. (Tübingen 1994) 517-528.

98 Auch hierzu Beyer, Sport $668 \mathrm{ff}$.

99 Eine Zusammenfassung der Reformpädagogik bei Größing, Reformen 654f.; siehe auch das ausführliche Zitat des Reformpädagogen Herman Nohl bei Wolfgang Buss, Das Göttinger IfL und die Diskussion über Sport und Sportlehrerausbildung an den Universitäten der britischen Zone 1945/46. Die Neuauflage einer Auseinandersetzung der Weimarer Zeit im Schatten der nationalsozialistischen Fehlentwicklung, in: Sozial- und Zeitgeschichte des Sports 1 (1987) 2, 68 f.; im folgenden zitiert: Buss, Das Göttinger IfL.

100 Buss, Das Göttinger IfL $66 \mathrm{ff}$.

$101 \mathrm{Vgl}$. Beyer, Sport 671. 
nem Zitat des ehemaligen Militärleistungssportlers Carl Krümmel ${ }^{102}$ belegen, der nicht nur die Funktion des Chefs des Amtes K, sondern auch die des Direktors eines besonderen Typus der IfL, der Führerschule des Berliner Hochschulinstituts für Leibesübungen im Schloß Neustrelitz, ausübte. Dort sammelten sich vor Abschluß der einjährigen Pflichtsportausbildung alle männlichen Studierenden zu einem vierwöchigen Prüfungslager, das als geländesportliche Lagergemeinschaft zu bezeichnen ist und dessen Teilnehmer von Krümmel mit folgenden Worten begrüßt wurden:

"Ich möchte gern den Turnlehrer verschwinden lassen dadurch, daß ich sämtliche Lehrer zu Leibeserziehern mache. Der Nationalsozialismus verlangt die totale Erzieherpersönlichkeit [...]. Erzieher werden sollen tüchtige, frische, aktiv in den Gliederungen der Bewegung stehende, Soldat gewesene und felddienstfähige Männer. [...] Im Sport herrscht der nackte, klare, brutale Wille, wobei ,brutal' noch eine Zärtlichkeitsbezeichnung ist. ${ }^{\text {" }} 103$

Ein Blick auf die Inhalte der, Grundausbildung' zeigt, daß der Theorieanteil verschwindend gering war: in 16 Semesterwochenstunden lediglich ein Fünftel Theorie $^{104}$. Krümmel hatte zwar alle Hochschulen und Universitäten mit Instituten für Leibesübungen und zahlreichem Personal besetzt, aber aus dem Universitätsverband herausgelöst. So konnte er die IfL zu ihm unmittelbar unterstellten Instituten machen, deren Leiter nicht mehr Hochschulprofessoren, sondern Oberregierungsräte waren ${ }^{105}$.

Dieses Beispiel enthält also folgende allgemeine Aussage: Sofern noch Sportwissenschaft getrieben wurde, geschah dies als Militärmedizin wie in Hamburg, an dessen IfL seit 1934 die ersten sportwissenschaftlichen Promotionen in Deutschland möglich waren ${ }^{106}$. Wenn man bedenkt, daß - wie in Göttingen - die sportpraktischen Kurse mittwochs und freitags von 6 bis $8 \mathrm{Uhr}$ morgens stattfanden ${ }^{107}$, dann dürfen wir unser Zwischenergebnis auch an die Memoiren des Münsteraner Mathematik-Professors Behnke anknüpfen:

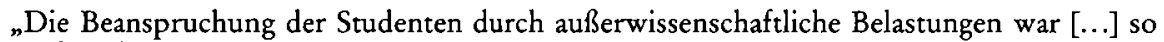
groß, daß normale Vorlesungen kaum zustande kamen. [...] Während eines großen Teils des Tages waren die Studenten durch politische Verpflichtungen eingespannt. Sie liefen plötzlich aus der Vorlesung weg, weil sie zum befohlenen Mittagessen in der Mensa sein mußten, sie schliefen morgens auf Bänken ein, weil sie seit fünf Uhr morgens oder gar während der ganzen Nacht an Übungen [...] teilgenommen hatten. Kamen sie noch in die gekürzten Vorlesungen, so fanden sie aber unmöglich Zeit, noch ein Buch zu lesen. So lag die Ausbildung der Studenten völlig darnieder. ${ }^{\text {108 }}$

102 Zu Krümmel als Sportler Ueberborst, Carl Krümmel $11 \mathrm{ff}$.

103 Zit. n. Hajo Bernett, Wissenschaft und Weltanschauung. Sportlehrerausbildung im Dritten Reich, in: Ursachen der Schulsportmisere in Deutschland, hrsg. v. Arnd Krüger, Dieter Niedlich (London 1979) $39 \mathrm{ff} . ;$ im folgenden zitiert: Bernett, Wissenschaft.

104 Bernett, Wissenschaft 35.

105 Carl Diem, Mein Leben 153.

106 Michael Joho, Prof. Dr. Wilhelm Knoll und die Entwicklung der Sportwissenschaft in Hamburg bis 1945, in: Stadion XII/XIII (1986/7) $273 \mathrm{ff}$.

107 Buss, Das Göttinger IfL 74.

108 Zit. n. Buss, Peiffer, Hochschulsportforschung 50. 
Anstelle der Weimarer Vorstellung der, universitas litterarum' trat im Nationalsozialismus ein aus Hitlers Rede vom ,freien, herrlichen Raubtier' abgeleitetes Wissenschaftsverständnis ${ }^{109}$, das beispielsweise Friedrich Stünzner in seiner $\mathrm{Me}$ thodik der Leibeserziebung im Schulunterricht von 1938 so formuliert: „Unter dem Totalitätsanspruch des Nationalsozialismus ist die Erziehung des Leibes und durch den Leib als politische Leibeserziehung zu einem unentbehrlichen Bestandteil der Gesamterziehungsgrundsätze unserer Zeit geworden." ${ }^{110}$ Im Kommentar der Richtlinien für die Leibeserziehung an Jungenschulen von 1937 heißt es entsprechend, daß „die Leibeserziehung Fundament der gesamten Erziehung“ sei und die "Erziehung des jungen Menschen von der körperlichen Erziehung“111 ausgehen müsse.

In der Absicht, die Gesinnung, Wehrkraft und Wehrwillen zu stärken, hatte Wissenschaft im traditionellen Verstande Humboldts keinen Platz. Während die Kontinuität darin bestand, daß der Hochschulsport autoritäre, revanchistische und antidemokratische Tendenzen ${ }^{112}$ der Weimarer Zeit fortsetzen konnte, lag die Diskontinuität darin, daß man die Reputation eines Faches durch Reduktion seiner wissenschaftlichen Ansprüche zu heben suchte. Martin Boye, Abteilungsleiter am IfL Berlin, sagte in diesem Sinne:

„Die Anwendung wissenschaftlicher Kategorien auf die Leibesübungen und die Schaffung eines Wissenschaftsgebäudes der Leibesübungen können möglich und für den philosophisch denkenden Menschen interessant sein; praktisch sind sie von minderer Bedeutung in einer Zeit, die tatkräftiges Handeln und persönlichen Einsatz verlangt." "113

Es ist das Dilemma (und auch die Tragik) des Idealismusparadigmas, daß sowohl diese Diskontinuität wie jene Kontinuität aus einem gemeinsamen Ursprung in der Lebensphilosophie ${ }^{114}$ abgeleitet werden können, der über Nietzsche, Spranger und Dilthey zu einer die Sportwissenschaft nach 1930 beherrschenden

109 Buss, Peiffer, Hochschulsportforschung 43, 46; weitere Zitate und einen zusammenfassenden Kommentar bietet Robert Probl, Grundriß der Sportpädagogik (Frankfurt a.M. 1999) $59 \mathrm{ff}$.

110 Friedrich Stünzner, Methodik der Leibeserziehung im Schulunterricht (Leipzig 1938) V.

111 Zit. n. Joch, Sport 703; dort auch weitere Zitate.

112 Vgl. Teichler, Der Weg Carl Diems 46; Armin Ader, Subjektive Wahrnehmung und historische Rekonstruktion, in: Sozial- und Zeitgeschichte des Sports 11 (1997) 1, 60ff.; Winkler, Weimar, $607 \mathrm{ff}$. Die Nationalsozialisten nahmen allerdings eine ambivalente Haltung zum Sport ein. Joch, Sport $701 \mathrm{f}$., $711 \mathrm{ff}$, weist daraufhin, daß ihr Begriff vom Sport zwischen Massenbewegung und Leistungsstreben lavierte, und faßt unter Berufung auf den Punkt 21 des von Hitler am 24. Februar 1920 vorgestellten Parteiprogramms der NSDAP (damals D.A.P.) zusammen, daß „seit Beginn ihrer Bewegung die Nationalsozialisten bemüht waren, sich als Förderer des Sports darzustellen “, weil sie Turn- und Sportabteilungen zu Tarnungszwecken benutzten. Bernett, Der deutsche Sport im Jahre 1933, $226 \mathrm{f}$., betont die nationalsozialistische Kritik der Olympischen Spiele, die eine ",französische Schöpfung““ seien und an die Beschämung von 1932 erinnern, ",als ein wollhaariger Neger mit Wulstlippen in Los Angeles den deutschen Meisterläufer besiegte ${ }^{\star}$.

113 Zit. n. Buss, Das Göttinger IfL 72.

114 Zur immanenten Widersprüchlichkeit der Lebensphilosophie Lieber, Kulturkritik VII. 
„Biologie der Leibesübungen ${ }^{{ }_{115}}$ und Baeumlers Rede vom Leib als „Politicum "116 führt. Ein solcherart verstandener Sport, der auf die Unvereinbarkeit von biologistischem und demokratischem Denken weist ${ }^{117}$, konnte zur Rechtfertigung der ,Auslese' herangezogen werden, d.h. der Integration derjenigen, die physisch, rassisch und politisch geeignet erschienen, im Sinne der nationalsozialistischen Ideologie zu handeln, und dem Ausschluß derjenigen, die dafür als untauglich galten ${ }^{118}$. Indem die Sportwissenschaft im Nationalsozialismus von der Sportwissenschaft der Weimarer Zeit die formale Etablierung und quantitative Ausgestaltung übernahm, aber durch eine spezifische inhaltliche Organisation umfunktionierte, konnte das humanistische Menschenbild durch das des ,politischen Soldaten' ersetzt werden ${ }^{119}$.

\subsection{Diem und die Reichsakademie für Leibesübungen (RfL)}

Um nachvollziehen zu können, inwieweit als einigende Klammer in den nun folgenden Überlegungen zur Reichsakademie für Leibesübungen (RfL) und zu den Olympischen Spielen von 1936 Carl Diems Rolle im Deutschen Reichsbund für Leibesübungen (DRL) zu bedenken ist, wird ein kurzer Blick auf die Vorgänger-

115 Edmund Neuendorff, Geschichte der neueren deutschen Leibesübung vom Beginn des 18. Jahrhunderts bis zur Gegenwart, Bd. 4 (Dresden o. J.) 690.

116 Zit. n. Volker Caysa, Nietzsches Leibphilosophie und das Problem der Körperpolitik, in: Nietzsche-Forschung. Ein Jahrbuch, Bd. 4 (Berlin 1998) 285; vgl. zur Beziehung zwischen Nietzsche und Baeumler ausführlicher ebd. 285ff; zu Baeumler ferner Joch, Sport 703, 714ff; Leske, Philosophen, passim.

117 Vgl. Hans Fenske, Politisches Denken im 20. Jahrhundert, in: Politische Theorien von der Antike bis zur Gegenwart, hrsg. v. Hans Joachim Lieber (Bonn 1991) 809: „Die von den Sozialdarwinisten entwickelten Vorstellungen konnten mit Demokratie kaum zusammengehen, sollte das Individuum doch straff in die Gemeinschaft eingegliedert und in zentralen Entscheidungen seines Lebens von übergeordneten Instanzen abhängig gemacht werden “; vgl. ferner Hans-Günther Zmarzlik, Der Sozialdarwinismus in Deutschland als geschichtliches Problem, in: Vierteljahrshefte für Zeitgeschichte 11 (1963) $265 \mathrm{ff}$.; Grupe, Pädagogische Theorie 38. Damit hängt zusammen, daß auch Werte wie Achtung, Rücksicht und Fairneß, die das demokratische Ideal des Sports ausmachen, in der NS-Propaganda aufgegeben wurden; vgl. Joch, Sport 705: „Durch die Negierung derjenigen Wertordnung, die im Sport traditionell vorhanden war, dokumentiert sich eigentlich die Unvereinbarkeit von NS-Ideologie und Sport - trotz des Ansehens, das er genoß, und seiner großzügigen Förderung durch den NS-Staat. Ansehen und Förderung aber bezogen sich auf einen Sport, der seiner traditionellen Werte und seines traditionellen Ethos beraubt wurde. Was blieb, war eine seines Gehaltes entleerte Worthülse, die nun mit Inhalten beliebig gefüllt werden konnte, mit Inhalten, die den nationalsozialistischen Machthabern genehm waren und die ihren Machtanpruch zu sichern versprachen."

118 Vgl. Grupe, Pädagogische Theorie 78; Buss, Peiffer, Hochschulsportforschung $45 \mathrm{ff}$. Im Sinne einer historischen Kontinuität des Sozialdarwinismus vgl. das Zitat von Diem, Ausgewählte Schriften, Bd. 2, 137, aus dem Jahre 1919: „Es heißt jetzt zuerst einmal die Gesunden stark machen und nicht wie bisher das Mitleid mit den Kranken und Gebrechlichen erstikkend vor die Erfüllung dieser Aufgabe stellen!“

119 Vgl. Buss, Peiffer, Hochschulsportforschung $45 \mathrm{ff} .$, Bernett, Wissenschaft 33; Größing, Reformen 655. 
institution des DRL nötig: den Deutschen Reichsausschuß für Leibesübungen (DRA). Der DRA entstand 1917 aus dem DRAfOS (Deutscher Reichssausschuß für Olympische Spiele) und war die parlamentarisch-demokratische Dachorganisation der bürgerlichen Turn- und Sportvereine mit Diem als Geschäftsführer ${ }^{120}$.

Als im Frühjahr 1933 Carl Diem gerüchteweise hört, daß er „im Kampf um den einzuberufenden Reichskommisar" in "erster Linie“ genannt werde, bewirbt er sich auf Anraten seines Sportkameraden Walther von Reichenau, dem Chef des Ministeramtes im Reichswehrministerium, um diesen Posten. Diem trägt am 25. März 1933 im Reichsarbeitsministerium, in dessen Bereich der Sport zunächst zu fallen scheint, einen Programmentwurf vor, der nach seinen eigenen Worten lediglich das alte Konzept des DRA wiederholt. Diem hält an der Idee der eingeschränkten Subsidiarität fest, „daß der Sportbetrieb selbständig und privat bleiben sollte, dafür aber systematisch vom Staate zu fördern sei“, ersetzt aber hinsichtlich ihrer Verwirklichung das demokratische Prinzip des DRA der Weimarer Zeit durch eine autoritär-diktatorische Leitung. So heißt es beispielsweise über das Vereinsleben:

„Ziel: die freien Kräfte des Volkslebens in einheitliche Richtung bringen. Überfeinerung, Ziellosigkeit, Materialismus, Zersplitterung beseitigen. Nationale Durchglühung des Sports. Methode: lose Zügelführung bei Gutwilligen, Rücksichtslosigkeit bei Widerspruch und Faulheit. Führerwechsel, wo notwendig. ${ }^{\text {"121 }}$

Diem aber unterschätzte zum einen die Vorbehalte der Nationalsozialisten gegen seine Funktion als herausragender Präsentant des bürgerlichen Sports. In der NSPresse wurde z.B. das schlechte Abschneiden der deutschen Mannschaft bei den Olympischen Spielen 1932 in Los Angeles mit dem Vorwurf verknüpft, daß er selbst 1. Klasse, die Mannschaft jedoch nur Touristenklasse gereist sei. Zum anderen rechnete Diem nicht mit der Radikalität der SA, die keineswegs, wie von ihm erhofft, ihre Sportanstalten dem DRA unterstellen wollte, aber auch nicht mit der Bereitschaft der eigenen Fachverbände zur raschen Unterwerfung ${ }^{122}$. Am 28. April 1933 wird überraschend der für die Fachwelt völlig unbekannte Führer der SA-Gruppe Mitte, Hans von Tschammer und Osten, vom Innenminister zum Reichssportkommissar und am 21. Juli 1933 zum Reichssportführer ernannt, wobei sogar gemunkelt wurde, man habe ihn mit seinem weit sportlicheren älteren Bruder verwechselt ${ }^{123}$.

120 Vgl. Hajo Bernett, Erich Beyer, Deutsche Hochschule für Leibesübungen (DHfL), in: Sportwissenschaftliches Lexikon, hrsg. v. Peter Röthig u. a. (Schorndorf 61992) 109; im folgenden zitiert: Beyer, Bernett, DHfL; vgl. Beyer, Sport $659 \mathrm{ff}$.

121 Zit. n. Bermett, Der deutsche Sport im Jahre $1933229 \mathrm{f}$. Eine völlig unkritische Deutung dieses progammatischen Übergangs vom DRA zum DRL bei Christiane Eisenberg, „English sports ${ }^{\star}$ und deutsche Bürger: Eine Gesellschaftsgeschichte 1800-1939 (Paderborn 1999) 441; im folgenden zitiert: Eisenberg, "English sports“. Wir kommen darauf in der Anm. $145 \mathrm{zu}-$ rück.

122 Bernett, Der deutsche Sport im Jahre 1933 230; vgl. Teichler, Die Rolle Carl Diems 57-61. 123 Vgl. Bernett, Der deutsche Sport im Jahre 1933 231; Teichler, Der Weg Carl Diems 64. Die Gründe für die Ernennung von Tschammers liegen nach Eisenberg, „English sports“ 390 , 
Obgleich von Tschammer am 10. Mai 1933 den DRA auflöste, agierte er danach sehr vorsichtig. Er verzögerte die Liquidation des DRA bis 1934, und an der Gründungssitzung des DRL als ,Rechtsnachfolger' des DRA am 9. März 1934 nahmen außer Tschammers eigenen Leuten ausschließlich ehemalige DRA-Funktionäre teil. Von besonderer Bedeutung war hier der erfahrene Organisator Diem, dessen Vertrag verlängert und der mit dem Organisationssausschuß zum Aufbau des DRL beauftragt wird ${ }^{124}$.

Diese Aufgabe jedoch konnte den ehrgeizigen Diem nicht ausfüllen. Im Zuge der Auflösung des DRA war Diem nicht nur aus seinem Amt als Generalsekretär des DRA, sondern auch unter nie ganz geklärten Umständen ${ }^{125}$ am 1. Mai 1933 als Prorektor der Deutschen Hocbschule für Leibesübungen (DHfL) entlassen worden. Bleiben konnte im Amt des Rektors der Jahre 1932/33 hingegen der Chirurg Ferdinand Sauerbruch, der zufälligerweise im I. Weltkrieg Hans von Tschammer und Osten behandelt hatte und bereits 1932 mit markigen Worten betonte, „daß die Jugend lernen müsse zu gehorchen, den Mund zu halten, Autorität anzuerkennen und auf die Eigenart der Person zu verzichten ${ }^{\star 126 .}$

Die in Berlin-Spandau gelegene DHfL war nach einem Beschluß des DRA vom 3. Oktober 1919 am 15. Mai 1920 in Anwesenheit des Reichspräsidenten Friedrich Ebert als private, aber vom Staat finanzierte Einrichtung des DRA zur sechssemestrigen Ausbildung freiberuflicher Sportlehrer und -lehrerinnen eröffnet worden ${ }^{127}$. Der Z weck von Diems interdisziplinärem Konzept ${ }^{128}$ - wie es im $\$ 1$ der Satzung von 1922 heißt -, das „Gebiet der körperlichen Erziehung wissenschaftlich und praktisch zu erforschen und das Verständnis für Leibesübungen in allen Kreisen des deutschen Volkes zu vertiefen" 129 , spiegelt sich auch in den Lehrkräften der DHfL; man denke nur an den Rektor, den renommierten Chirurgen August Bier, oder Eduard Spranger, den „außerordentlichen“ Lehrer in der Abteilung „Erziehungslehre"130.

zum einen in organisatorischen Erfordernissen (Zugehörigkeit des Sports zum Innenministerium) und zum anderen in von Tschammers Sprachkenntnissen und Umgangsformen, die ihn für das Ausland repräsentabel machten.

$124 \mathrm{Vgl}$. Hajo Bernett, Die Reichsakademie für Leibesübungen im Traditionsverständnis der deutschen Sporthochschule, in: Stadion XVIII, 2 (1992) 249; im folgenden zitiert: Bernett, Reichsakademie; Bernett, Umbruch $18 \mathrm{f}$.

125 Nach Carl Diem, Mein Leben 95, sei der Grund für seine Entlassung seine ablehnende Haltung gegenüber Wehrsportübungen gewesen.

126 Teicbler, Der Weg Carl Diems 53; vgl. Bernett, Der deutsche Sport im Jahre 1933 235; zu Einzelheiten des Vertrags Teichler, Die Rolle Carl Diems 62, Anm. 23.

127 Vgl. Bernett, Der deutsche Sport im Jahre 1933 235; Bernett, Reichsakademie 247; Beyer, Bernett, DHfL; Beyer, Sport $669 \mathrm{f}$.

128 In seiner Denkschrift über die Gründung einer Deutschen Hochschule für Leibesübungen aus dem Jahre 1919 betont Diem, Ausgewählte Schriften, Bd. 2, 138 f., daß der „Wiederaufbau unserer Körperkraft und Gesellschaft“ eine „Sammelstelle“ zur Bündelung sportwissenschaftlicher Erkenntnisse erforderlich mache.

129 Carl-Diem-Institut, Dokumente 123.

130 Carl-Diem-Institut, Dokumente 127. 
Nach seiner Entlassung aus dem Prorektorat verfolgte Diem mehrfach Pläne, die DHfL aus der freien Trägerschaft in eine zentrale Reichshochschule für alle Sportlehrer zu überführen, zuletzt im Juni 1939, reüssierte jedoch nicht ${ }^{131}$. Im Sommersemester 1933 überträgt - wohl auch auf studentischen Wunsch hin Reichsminister Rust Alfred Baeumler einen Lehrauftrag für die DHfL und sogar die Verantwortung für Forschung und Lehre; Diem zufolge lichteten sich jedoch die Reihen seiner Hörer sehr schnell, und Baeumlers Interregnum dauerte nur kurz. Erst mit Führererlaß vom 7. April wird die ehemalige DHfL als Reichsakademie für Leibesübungen nachträglich zu einer ,Reichsbehörde‘ unter dem Direktor Krümmel erklärt, der ähnlich wie in Neustrelitz der Akademie ein wehrsportliches Gepräge gibt. Die RfL stellt mit Beginn des II. Weltkriegs ihren Betrieb ein 132 .

Obgleich die DHfL und ihre Vorgängerinstitutionen, das Dresdener Sportlaboratorium 1911 und das seit 1906 verfolgte Stadion-Projekt in Grunewald, von Beginn an im Spannungsfeld von Zwecklosigkeit und Zweckdeterminiertheit stehen ${ }^{133}$, wird ein großer Kontrast zwischen Diems Konzept freier Wissenschaft für die DHfL aus dem Jahre 1922 und dem Wissenschaftsverständnis der RfL vor allem in der Abteilung „Erziehungslehre“ ersichtlich. In ihm wird anstelle von Sprangers Erziehungslehre die ,politische Leibeserziehung' Baeumlers vertreten, und es liegt in der Konsequenz dieses Traditionsbruches, daß bis Mitte 1938 nur 700 Lehrer, aber 10000 Übungsleiter die Akademie frequentierten ${ }^{134}$. Da Diem in seinen Konzepten aber stets die ideelle Kontinuität zwischen DHfL und RfL hervorgehoben hatte, darf man vermuten, daß es für ihn „keinen Bruch zwischen einer bürgerlich-demokratischen und einer nationalsozialistisch-autoritär geführten Institution gab“135. Klemperer bemerkte dagegen über die Gründung der RfL:

131 Vgl. Bernett, Reichsakademie 251.

132 Vgl. Bernett, Wissenschaft 36f.; Bernett, Der deutsche Sport im Jahre $1933235 \mathrm{ff}$.

$133 \mathrm{Vgl}$. Court, Interdisziplinäre Sportwissenschaft $36 \mathrm{f}$.

134 Vgl. Bernett, Wissenschaft 39; Bernett, Reichsakademie 250.

135 Bernett, Reichsakademie 251; vgl. Eisenberg, „English sports“ 405, 441. Vgl. die Ausführungen Diems von 1947 über „Aufgaben, Unterricht und Ziele“ der in diesem Jahr in Köln eröffneten Sporthochschule (DSHS), die er durch einen Vergleich mit der DHfL des Gründungsjahres 1920 einleitet: „Ihr Lehrkörper wurde der philosophischen und medizinischen Fakultät der Universität Berlin entnommen; es wurden Forschungslaboratorien für Physiologie und Psychologie der Leibesübungen eingerichtet und so Lehre wie Forschung organisch mit der Universität verbunden. [...] In Deutschland übernahmen die Universitäten nach dem Vorbild der Berliner Hochschule allmählich die Sportlehrerausbildung. [...] In dieser (!) Entwicklungszeit brach der Nationalsozialismus ein, der die Deutsche Hochschule für Leibesübungen sofort aufhob und unter Verwendung ihrer Einrichtung und eines ihm gefügigen Teils des Lehrkörpers die Reichsakademie für Leibesübungen gründete. Er löste praktisch deren Beziehungen zur Universität, indem er die von ihm gewählten wissenschaftlichen nazistischen Lehrkräfte der Universität aufzwang, ohne damit ihre geistige Bedeutung zu erhöhen [...]. Wenn heute die [...] Institute innerhalb der Universität um ihre Anerkennung ringen müssen und es sogar Universitäten geben soll, die solche Institute ablehnen - als ob die Körpererziehung von der Wissenschaft der Pädagogik ausgenommen werden könne -, so ist dies die Folge jener nationalsozialistischen Zertrümmerung, deren Wirkungen auch an 
„Es gibt jetzt eine Hochschule für Sport, ein Sportakademiker ist jedem anderen Akademiker mindestens gleichgestellt - in den Augen des Führers sicherlich überlegen. ${ }^{“ 136}$

\subsection{Fazit: Zur wissenschaftlichen Bedeutung der Olympischen Spiele 1936}

Nachdem im Gefolge der Auflösung des DRA sämtliche Ambitionen Diems auf höhere Ämter gescheitert waren, beließ ihm von Tschammer die „sportfachliche Vorbereitung der XI. Olympiade ${ }^{{ }_{1137}}$. Dem Gegenstand dieser Studie gemäß muß ein Beispiel dafür genügen, wie Hitler sie als „Höhe-, aber auch [...] Abschlußpunkt der NS-Friedenspropaganda" 138 benutzte. Ausgerechnet am 1. August 1936, dem Tag der Eröffnungsfeier, legte das Heeresamt dem Oberbefehlshaber des Heeres eine Zuarbeit vor, die den Kriegsbeginn auf den 1. Oktober 1939 datierte und die jährlichen Rüstungskosten mit 9 Milliarden Reichsmark bezifferte ${ }^{139}$. Die Vermutung, daß ideelle und personelle Zusammenhänge zwischen den Olympischen Spiele von 1936 und dem „Kriegseinsatz der deutschen Geisteswissenschaften " 140 bestehen, führt vor dem Hintergrund des Idealismusparadigmas zur Formulierung der allgemeinen Aufgabe, zunächst nach möglichen Verbindunglinien zwischen der politischen Bedeutung der Olympischen Spiele von 1936 und Diems spezifischem Verständnis von Sportwissenschaft zu fragen. Für Diem bedeutet Sport eine nach Verwirklichung drängende, den ganzen Menschen in seiner Schönheit erfassende Kunst ${ }^{141}$, und Sportwissenschaft ist daher im Sinne

den Gelehrten nicht spurlos vorübergegangen sind“ (Diem, Ausgewählte Schriften, Bd. 2, $316 f$.). Dazu paßt die These von Wolfgang J. Mommsen, Wandlungen der nationalen Identität, in: Die Identität der Deutschen, hrsg. v. Werner Weidenfeld (Bonn 1983) 172: „Die Ausblendung der geschichtlichen Dimension aus dem Selbstverständnis der Bürger der Bundesrepublik Deutschland ist in den fünfziger und sechziger Jahren sehr ausgeprägt gewesen.“ 136 Klemperer, LTI 243.

137 Diem, zit. n. Teichler, Die Rolle Carl Diems 62.

138 Hans Joachim Teichler, Die Olympischen Spiele 1936 - eine Bilanz nach 60 Jahren, in: Aus Politik und Zeitgeschichte (1996) 22; im folgenden zitiert: Teichler, Die Olympischen Spiele 1936; eine Zusammenfassung der politischen Bedeutung dieser Spiele bietet auch Kr ̈̈ger, Einführung Bd. 2, 125-136; zu den Olympischen Spielen im Sinne eines „Großdeutschen Olympia" (Carl Diem) vgl. Krüger, Nationalfest 142f; zur Tradition deutsch-akademischer Olympien und Deutscher Kampfspiele siehe Beyer, Sport $676 \mathrm{ff}$.

139 Vgl. Teichler, Die Olympischen Spiele 193622.

140 Hausmann, Ritterbusch. In diesem Zusammenhang wäre es zum Beispiel eine Aufgabe, Beziehungen zwischen Diem und dem Zeitungswissenschaftler Emil Dovifat nachzugehen. Nach Hajo Bernett, Sportpublizistik im totalitären Staat 1933-1945, in Stadion XI (1985) 280 , „erklärte der Berliner Ordinarius Dovifat den Läufer von Marathon, den Diem in einer Erzählung gefeiert hatte, zum ersten ,PK-Mann', d.h. zum Prototyp eines Kriegsberichterstatters in einer Propaganda-Kompanie der Wehrmacht ${ }^{*}$. Zur Bedeutung Dovifats in der ,Aktion Ritterbusch'vgl. Hausmann, Ritterbusch 274, 299, 308.

141 Vgl. Carl Diem, Körpererzichung bei Goethe (Frankfurt a.M. 1948) 468: „Der Schönheitstrieb bestimmt die Regeln des Sports, in ihnen steht unbewußt ein menschliches Ideal an Bau, Bewegung und Verhalten zum Ziel. Sport treiben heißt den Körper und den Menschen 
der aristotelischen techne eine Wissenschaft, die sowohl um die Dignität der Praxis als auch die Notwendigkeit ihrer bewußten Reflexion weiß ${ }^{142}$. Während wir in den vorhergehenden Kapiteln über den Sport als Wissenschaft von der Praxis gehandelt haben, geht es in bezug auf die Olympischen Spiele um ihre Leistung für die Praxis, die hier ihre Vollendung findet.

Blicken wir nun auf die Grundthese zurück, daß die lebensphilosophische Berufung auf überzeitliche Ideale letztlich zu einer Preisgabe von Wissenschaft an subjektive Interessen und konkrete Mächte führt, wird die Problematik in Diems Verständnis von Sportwissenschaft offenbar: Seine platonistische Vorstellung einer Eigenwelt des Sports und das daraus entspringende Beharren auf der reinlichen Trennung von Sport und Politik ist nichts anderes als die sportwissenschaftliche Variante jenes lebensphilosophischen Dezisionismus ${ }^{143}$. Da in Diems Idee einer prinzipiell unzerstörbaren Sphäre des Sports als Kulturgut der Menschheit ${ }^{144}$ eine Instrumentalisierung durch Politik sui generis nicht vorkommen kann, tritt das bloße Organisationstalent an die Stelle der Einsicht in die Notwendigkeit einer kritischen Reflexion der Beziehung von Sport und Politik und seiner institutionellen Absicherung ${ }^{145}$ : „Wer die Politik aus dem Sport ausschalten will, handelt bereits politisch. [...] Es ist also ein leeres Geschwätz, zu sagen, die Politik gehöre nicht in den Sport. Ohne Politik gibt es keinen Sport."146

formen, Sport lehren heißt die Schüler zu einer bestimmten Idealgestalt und Idealgesinnung hinführen [...]. Wir können dessen innere Strebung nicht besser als unter dem Gesamtbegriff der Schönheit ausdrücken, was auch die unmittelbare Nachbarschaft und Verwobenheit mit jenem Schönheitsstreben im engeren Sinne bestätigt, das wir Kunst nennen.“

142 Vgl. Friedrich Ernst Daniel Schleiermacher, Ausgewählte pädagogische Schriften (Paderborn 31983) 40: „Ist doch überhaupt auf jedem Gebiete, das Kunst heißt im engeren Sinne, die Praxis viel älter als die Theorie, so daß man nicht einmal sagen kann, die Praxis bekomme ihren bestimmten Charakter erst mit der Theorie. Die Dignität der Praxis ist unabhängig von der Theorie; die Praxis wird nur mit der Theorie eine bewußtere." Ausführlich Theodor Litt, Führen oder Wachsenlassen (Stuttgart 1965) $101 \mathrm{ff}$.

143 Court, Interdisziplinäre Sportwissenschaft 222.

${ }_{144}$ Vgl. Carl Diem, Wesen und Lehre des Sports und der Leibeserziehung (Berlin u. a. ${ }^{4} 1964$ ) 30: Vor 150 Jahren entwickelte sich der „moderne Sport zu einer das Leben mitprägenden Kulturerscheinung, die heute alle zivilisierten Völker ergriffen, die es bis zu einem gemeinsamen Hochfest der Welt gebracht hat."

$145 \mathrm{Vgl}$. Weingart, Einheit 24ff; Henning Krauß, Victor Klemperer und das „weitmaschige Wort Kulturkunde", in: lendemains 21 (1996) 124.

146 Bruno Malitz (1933); zit. n. Joch, Sport 736. Vor diesem Hintergrund zeugt die Hauptthese von Eisenberg, „English sports“ 393 , 441, beim vom DRL organisierten Sport habe es sich um einen „,reinen“ und „sachadäquaten “ Sport gehandelt, für einen Historiker von einer erstaunlichen Naivität. Gegen diese These, ausgerechnet der Nationalsozialismus habe den Eigenweltcharakter des Sports ermöglicht, zutreffend Joch, Sport 702. Teichler, Die Olympischen Spiele 1936 22, spricht von der „Fiktion eines unpolitischen Sports“ und einem "gigantischen Betrugsmanöver ${ }^{\alpha}$. Eine detaillierte Widerlegung der Thesen Eisenbergs. Jetzt bei Jürgen Court, Zur Renaissance des Idealismus - Bemerkungen zu Christiane Eisenberg, in: Transformationen des deutschen Sports seit 1939, hrsg. v. Michael Krüger (Hamburg 2001) $57 \mathrm{ff}$. 
Klemperes Tagebucheintrag vom 13. August 1936 „Mir ist die Olympiade so verhaßt, weil sie nicht eine Sache des Sports ist [...], sondern ganz und gar ein politisches Unternehmen"147, bedeutet daher im Lichte dieser Überlegungen, daß „niemand Gewissen hat als der Betrachtende“ ${ }^{148}$.

147 Klemperer, Tagebücher 1933-1941 293.

148 Johann Wolfgang von Goethe, Maximen und Reflexionen, in: Goethes Sämtliche Werke, Jubiläumsausgabe in 40 Bänden, Bd. 4 (Stuttgart, Berlin o. J.) 212. Das vollständige Zitat "Der Handelnde ist immer gewissenlos, es hat niemand Gewissen als der Betrachtende" variiert Klemperer, Tagebücher 1945-1949 233: „Nur der Betrachtende hat recht“ (Tagebuch vom 24. April 1946). 